\title{
Effect of Johne's disease status on reproduction and culling in dairy cattle
}

\author{
R. L. Smith, ${ }^{* 1}$ R. L. Strawderman, † Y. H. Schukken,ł S. J. Wells,§ A. K. Pradhan,ł L. A. Espejo,§ R. H. \\ Whitlock,\# J. S. Van Kessel, || J. M. Smith, ग D. R. Wolfgang, ${ }^{* *}$ and Y. T. Gröhn* \\ *Section of Epidemiology, Department of Population Medicine and Diagnostic Sciences, College of Veterinary Medicine, \\ †Department of Biological Statistics and Computational Biology, and \\ $\ddagger$ Quality Milk Production Services, Department of Population Medicine and Diagnostic Sciences, College of Veterinary Medicine, \\ Cornell University, Ithaca, NY 14850 \\ §Department of Veterinary Population Medicine, University of Minnesota, St. Paul 55108 \\ \#New Bolton Center, Department of Clinical Studies, School of Veterinary Medicine, University of Pennsylvania, Kennett Square 19348 \\ ||Environmental Microbial and Food Safety Laboratory, ANRI, USDA-ARS, Beltsville, MD 20705 \\ TDepartment of Animal Science, University of Vermont, Burlington 05405 \\ ${ }^{* *}$ Department of Veterinary and Biomedical Science, Penn State University, University Park 16802
}

\section{ABSTRACT}

Among the costs attributed to Mycobacterium avium ssp. paratuberculosis (MAP) infection in dairy cattle, the effects on reproduction and culling are the least documented. To estimate the cost of MAP infections and Johne's disease in a dairy herd, the rates of calving and culling were calculated for cows in each stage of MAP infection relative to uninfected cows. Data from 6 commercial dairy herds, consisting of 2,818 cows with 2,754 calvings and 1,483 cullings, were used for analysis. Every cow in each study herd was tested regularly for MAP, and herds were followed for between 4 and 7 yr. An ordinal categorical variable for Johne's disease status [test-negative, low-positive (low-shedding or ELISA-positive only), or high-shedding] was defined as a time-dependent variable for all cows with at least 1 positive test result or 2 negative test results. A Cox regression model, stratified on herd and controlling for the time-dependent infection variable, was used to analyze time to culling. Nonshedding animals were significantly less likely to be culled in comparison with animals in the low-shedding or ELISA-positive category, and highshedding animals had nonsignificantly higher culling rates than low-shedding or ELISA-positive animals. Time to calving was analyzed using a proportional rates model, an analog to the Andersen-Gill regression model suitable for recurrent event data, stratifying on herd and weighted to adjust for the dependent censoring caused by the culling effects described above. Highshedding animals had lower calving rates in comparison with low-shedding or ELISA-positive animals, which tended to have higher calving rates than test-negative animals.

\footnotetext{
Received September 17, 2009.

Accepted March 30, 2010.

${ }^{1}$ Corresponding author: rls57@cornell.edu
}

Key words: Johne's disease, reproduction, culling, survival analysis

\section{INTRODUCTION}

Johne's disease (JD) is a chronic disease of ruminants caused by intestinal infection with the pathogen Mycobacterium avium ssp. paratuberculosis (MAP). The pathogen is pervasive on US dairy farms, with approximately $68 \%$ of herds infected (USDA-APHIS-VSNAHMS, 2007). Infection with MAP typically occurs in calves (Clarke, 1997), which, after a period of transient shedding (van Roermund et al., 2007), then enter a latent, nonshedding stage of varying length. This latent stage is followed by a period of low and intermittent shedding of MAP with no obvious clinical symptoms (Whitlock et al., 2000). If left to progress, clinical JD may develop, with a high level of MAP shedding (Whitlock et al., 2000).

Johne's disease has been estimated to cost the US dairy industry $\$ 200$ to $\$ 250$ million annually (Ott et al., 1999). Two of the possible costs associated with JD are forced early culling and decreased reproductive performance. The primary economic factor in dairy production is undoubtedly milk production, but culling and reproduction are important secondary factors. Culling at the appropriate time allows for replacement of animals with animals of higher production potential. In contrast, culling too early can remove animals from the herd before reaching their production potential, resulting in an overall economic loss. Calving can improve milk production by initiating a new lactation, increasing milk production for a period of months. Reproduction also provides calves for sale or replacement. Both culling and reproduction may be affected by infectious diseases (Gröhn et al., 1998; Fourichon et al., 2000).

Animals with JD may be culled because of onset of clinical signs, such as diarrhea or wasting (Collins, 
2003). Animals may also be culled for decreasing milk production, which is associated with progression of JD (Smith et al., 2009). In addition, structured JD control programs often recommend immediate culling of highshedding animals to limit environmental contamination and the transmission of MAP to herd mates, particularly calves. Culling has been shown to be a potentially effective tool to reduce prevalence, but increased culling by itself is not expected to eliminate MAP infection from a dairy (Lu et al., 2008). In addition, a combination of imperfect diagnostic techniques and slow development of clinical symptoms often results in delayed culling or retention of low-shedding animals. On most farms, low-shedding animals are retained because of absence of clinical signs in these animals and the high cost of raising or purchasing replacements (Dorshorst et al., 2006).

It is hypothesized that clinical JD may cause a negative energy and protein balance, which decreases fertility in dairy cows. However, not all studies have found an association between JD and reproductive variables, and some have found an association in the opposite direction. One prospective cohort study found that ELISApositive animals had significantly higher numbers of days open, but that fecal culture-positive animals had nonsignificantly fewer days open (Johnson-Ifearulundu et al., 2000). Several older studies found that MAPinfected cows were culled younger (Buergelt and Duncan, 1978), culled more frequently for owner-reported infertility (Merkal et al., 1975), or experienced longer calving intervals (Abbas et al., 1983) than uninfected cows. A later longitudinal study did not find an association between MAP shedding and either calving success or early culling in seasonally bred herds (de Lisle and Milestone, 1989), although analysis of infertility was not the aim of that study and the observation was made using data collected for other purposes, meaning that reproductive issues were inferred rather than directly observed. A slightly more recent case control study also found no association between JD status and calving interval (McNab et al., 1991). A recent study even found that cows with a MAP ELISA-positive serum test spent fewer days in a nonpregnant state (Lombard et al., 2005). It is important to recognize that this may be a biased result because ELISA-positive animals may be culled earlier. With these conflicting results, biases and confounding must play a major role. One study has shown that subclinical MAP infection, as measured by ELISA positivity, may be associated with an increase in conception rates (Marcé et al., 2009), but this association was seen to decrease with increased age and parity. Another study found that pregnancy rate increased with MAP positivity if ELISA and fecal culture were considered in parallel or if the analysis was limited to ELISA-positive animals, but that limiting the analysis to animals positive by fecal culture resulted in a nonsignificant decrease in pregnancy rate (Gonda et al., 2007).

Studies of reproduction need to be carefully analyzed to control for proper time at risk in all animals. In particular, when the risk of censoring (culling) is related to both the risk of being ELISA-positive and the risk of the event (calving), care must be taken in the analyses to avoid biases caused by dependent censoring. This issue of dependent censoring was highlighted in a recent prospective study. Raizman et al. (2007) evaluated the effect of MAP infection on reproduction in dairy cattle and found that infected animals are less likely to conceive. Although this study employed standard time to event analysis to evaluate the effect of MAP status on reproduction, it still identified 2 potential biases that were not controlled. First, infected animals were bred fewer times than uninfected animals, thereby decreasing the probability of conception. Second, MAP-infected animals were culled from the herd early based on clinical JD. It has been previously shown that simulated positive dependence between culling and conception may bias the results of a proportional hazards model for time to conception (Allore et al., 2001). Because culling plays the role of a censoring variable in statistical models for calving, bias can also occur in the analysis of calving rates unless one properly controls for the interdependence between calving rates, culling rates, and JD.

The purpose of this study was to obtain estimates of the effect of JD on both time to culling and time to calving in dairy cattle, using recent advances in statistical methods for analyzing recurrent event outcomes (i.e., calvings) subject to dependent censoring (i.e., culling). The results can be applied to economic models considering the cost of JD to the dairy industry.

\section{MATERIALS AND METHODS}

These data are the combination of 2 data sets. The first data set is from of a longitudinal study on 3 dairy herds enrolled in an ARS-Regional Dairy Quality Management Alliance (RDQMA) study. In 2004, the RDQMA identified a commercial dairy herd in 3 of its member states (New York, Vermont, and Pennsylvania) to serve as longitudinal study herds. The details of this study have been described previously (Pradhan et al., 2009). Briefly, these 3 herds were visited on a quarterly basis to collect individual animal samples. During these visits all production, breeding, and health records were obtained through copying on-farm electronic records and through records obtained from the DHIA. Herds $\mathrm{A}$ and $\mathrm{C}$ used a synchronization program for reproduction only sporadically, and herd $\mathrm{C}$ bred some animals 
naturally. Serum samples were collected quarterly and fecal samples were collected biannually from each adult animal in each herd, and all samples were shipped overnight to The University of Pennsylvania Johne's Laboratory (New Bolton Center) for analysis (Pradhan et al., 2009). Serum samples were tested by the ParaChek (Prionics USA Inc., La Vista, NE; formerly CSL/Biocor) ELISA for antibody reactions to MAP antigens, for which sensitivity and specificity estimates for animals shedding MAP are 0.24 to 0.80 and 0.98 to 0.99 , respectively (Nielsen and Toft, 2008). Fecal samples were tested by 4 -tube fecal culture for presence of viable MAP organisms (Pradhan et al., 2009), and the total sum of colony-forming units across 4 tubes was multiplied by 5.3 to determine colony-forming units per gram, with estimated sensitivity and specificity of 0.7 to 0.74 and 1.0, respectively, for animals shedding MAP (Nielsen and Toft, 2008). Diagnostic results were reported as positive or negative for ELISA and as colony-forming units per gram for culture. Herd owners were informed of the results of all diagnostic tests and encouraged to cull animals with high-positive fecal cultures.

The second data set used was collected from herds in Minnesota participating in the Johne's Disease Demonstration Herd Project (JDDHP), which began in 2000 and has been described previously (Ferrouillet et al., 2009). Briefly, serum and feces were collected from all adult cows at the initiation of the study and once per year in following years, either upon confirmed pregnancy or at a single date. All monthly production, breeding, and culling records were obtained through the DHIA. For repeat breedings, herds D and E used a synchronization program and herd $\mathrm{F}$ used natural breeding. Serum samples and fecal samples were processed by the Minnesota Veterinary Diagnostic Laboratory (St. Paul). Serum samples were tested using an ELISA test kit for serum antibody detection (IDEXX Laboratories Inc., Westbrook, ME) as indicated by the test kit label, with estimated sensitivity and specificity for animals shedding MAP of 0.24 to 0.74 and 0.88 to 1, respectively (Nielsen and Toft, 2008). Fecal samples were tested for presence of viable MAP organisms using bacterial culture with Herrold's egg yolk media using 72 $\mathrm{h}$ of sedimentation (Wells et al., 2002), with estimated sensitivity and specificity for animals shedding MAP of 0.7 to 0.74 and 1.0, respectively (Nielsen and Toft, 2008). Diagnostic test results were reported as positive or negative for ELISA and as colony-forming units per tube for culture. Herd owners agreed to implement control measures that included limiting exposure of young stock to adult cows and testing to identify and remove or quarantine infectious animals.
For all cows, Johne's disease status was initially divided into 3 categories: test-negative, low-positive (lowshedding or ELISA-positive only), and high-shedding (defined below). At each time $t$, test-negative animals were defined as animals for which there were at least 2 diagnostic test results reported and for which all diagnostic tests were negative. Animals with only 1 diagnostic test, if the result was negative, were removed from the analysis because of the lack of diagnostic sensitivity. Animals were assumed infected if at least 1 diagnostic test had a positive result. Animals were defined as low-positive after a positive ELISA or fecal culture of less than $30 \mathrm{cfu} / \mathrm{g}$ (RDQMA) or $50 \mathrm{cfu} /$ tube (JDDHP). Fecal shedding has been shown to correlate with ELISA status, allowing ELISA-positive animals to be classified as low-positive (van Schaik et al., 2003; Nielsen, 2008). Sensitivity differences between test methods were assumed to be negligible for purposes of this analysis. Animals were classified as high-shedding upon detecting a fecal culture of at least $30 \mathrm{cfu} / \mathrm{g}$ or 50 $\mathrm{cfu} /$ tube, representing the midpoint of a previously defined moderate shedding level (Whitlock et al., 2000). After classification as high-shedding, animals remained in this category; this is consistent with the progression of disease observed in the study herds. For purposes of analysis, we thus considered JD status to be an ordinal categorical variable, $J D(t)$, in which test-negative animals are the referent group and high-shedding animals are to be compared with low-positive animals. This ordinal structure is intended to reflect disease transitions through time (e.g., no detectable JD, progressing to subclinical JD, progressing on to clinical JD). Animals shedding high levels of MAP presumably must first pass through a low-shedding phase (whether or not such a state was observed); hence, a comparison between these 2 status levels is both more natural and informative than is a direct comparison between high-shedding and test-negative animals.

The analyses of the relationship between time to culling and Johne's disease can be performed using standard methods for time-to-event analysis, such as Cox's proportional hazards model (Cox, 1972). However, because culling rates depend on other factors (e.g., parity, herd) in addition to JD status, care must be taken to obtain an accurate estimate of the effect of JD on calving rates. Here, we use methods described by Miloslavsky et al. (2004) to fit a proportional rates regression model that allows us to characterize the effect of JD on calving rates, a recurrent event, adjusting for dependent censoring by culling. The proportional rates regression model for recurrent events is described in Lin et al. (2000) and is directly related to the Andersen-Gill regression model (Andersen and Gill, 1982). 


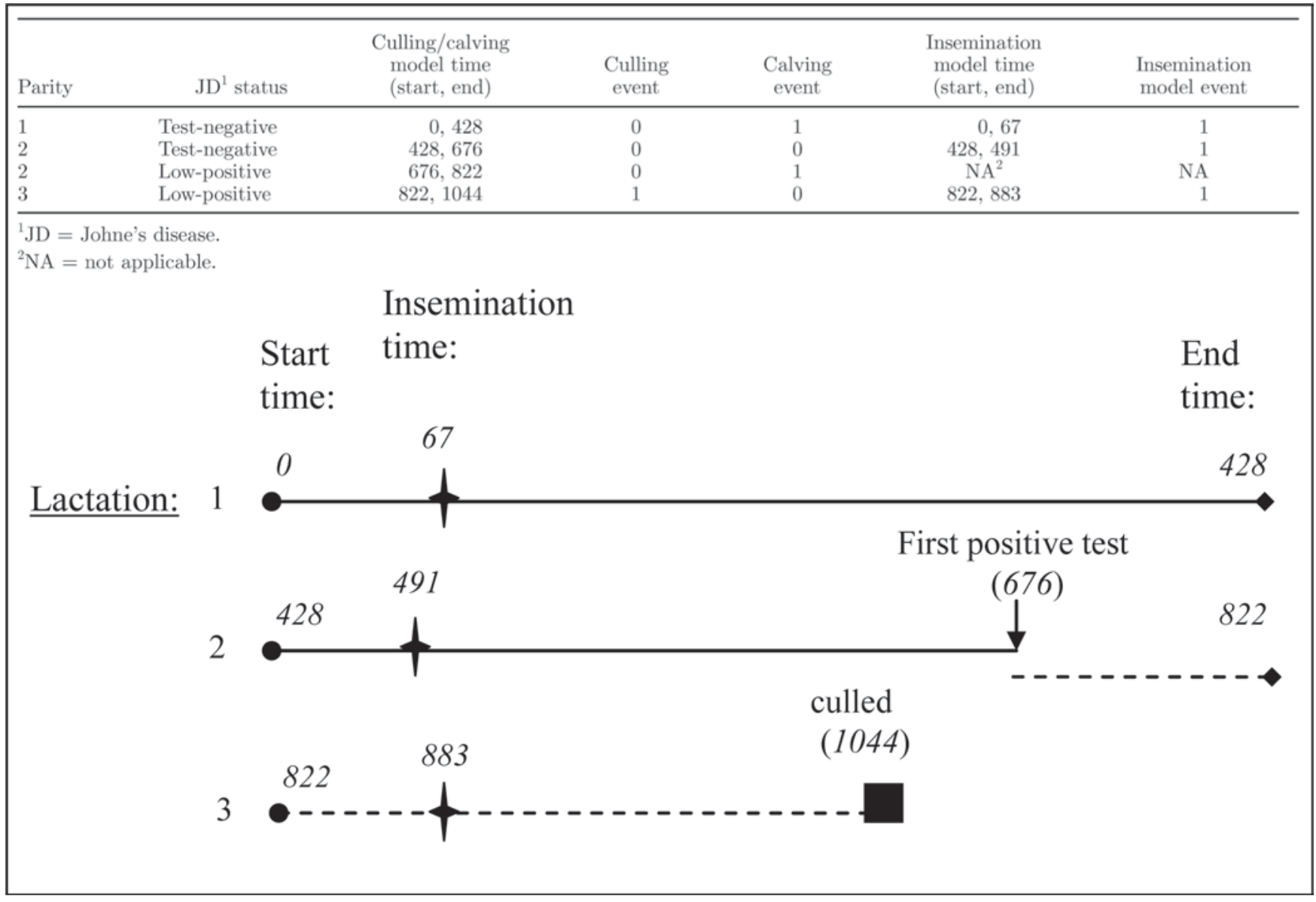

Figure 1. Diagram of datalines for the models for time to culling, calving, and insemination for a hypothetical animal. Solid circles indicate the start of a lactation, with the time in italics above being the start time for that lactation's first dataline. Stars indicate the first insemination during a given lactation. Diamonds indicate the end of a lactation, with the time in italics above. Arrows indicate positive test results, at which an animal is censored for the current dataline and a new dataline is started with the number in italics. The solid square in the third lactation indicates culling, with the time in italics above. Johne's disease status is indicated by the line, with solid lines indicating nonshedding status and dashes indicating Mycobacterium avium ssp. paratuberculosis shedding. The chart at the top shows the data that would be used in fitting each of the models.

\section{Culling}

First calving and culling dates were reported for each animal by their respective herd managers. Time to culling was calculated as the number of days between first calving and culling; 22 animals were removed from the data set because they were culled before their first calving. For each animal, this time was divided into separate records for each lactation, as shown in Figure 1, with time-at-risk in that parity beginning at the recorded calving date and ending with censoring at the following calving date when the animal is retained in the herd. If the animal was diagnosed antemortem, the record for the parity in which it was diagnosed was censored at the first positive test and a new record was created for the animal in the JD status category.
A Cox regression model was used to analyze time to culling; data were stratified by herd and lactation was included as a fixed effect. This regression model relates time to culling to these covariates through the following hazard function:

$$
\begin{aligned}
\lambda(t)= & \lambda_{0 H}(t) \exp \left[\beta_{1} J D_{1}(t)+\beta_{2} J D_{2}(t)+\beta_{3} P_{2}(t)\right. \\
& \left.+\beta_{4} P_{3}(t)+\beta_{5} P_{4}(t)+\beta_{6} P_{5}(t)\right]
\end{aligned}
$$

where $\lambda(t)$ is the hazard of culling at time $t$; $t$ is the age in days; $\lambda_{0 H}$ is an arbitrary and unspecified baseline hazard function for the given herd; $J D_{1}(t)$ is a binary variable indicating that the animal is low-positive at time $t ; J D_{2}(t)$ is a binary variable indicating that the animal is shedding high levels of MAP at time $t ; P_{i}(t)$ is 
a binary variable indicating that an animal is in parity $i$ at time $t ; \beta_{1}$ and $\beta_{2}$ are the parameters associated with animals in the low-positive and high-shedding JD categories, respectively; and $\beta_{3}$ through $\beta_{6}$ are the parameters associated with each parity (where parity 1 is the baseline). The variable $P_{5}(t)$ captures all animals having a parity of 5 or more. Because almost all censoring in this analysis occurs as a result of animals remaining under observation until the end of the study, it is reasonable to assume that censoring occurs independently of culling for the purposes of this analysis. Importantly, the effects of JD and parity are both coded as ordinal categorical variables; thus, for example, while the regression coefficient $\beta_{1}$ continues to measure the change in risk that occurs in moving from the testnegative referent group to the low-positive group, the regression coefficient $\beta_{2}$ measures the change in risk that occurs in moving from the low-positive group to the high-shedding group (i.e., as opposed to measuring the change in risk relative to the referent group). A similar interpretation applies to each of the regression coefficients for parity.

To account for a possible synergistic effect between parity and JD status, a categorical variable, $J P(t)$, was also created. This is an interaction indicating parity $>3$ and positive for MAP. This resulted in the following hazard function:

$$
\begin{gathered}
\lambda(t)=\lambda_{0 H}(t) \exp \left[\beta_{1} J D_{1}(t)+\beta_{2} J D_{2}(t)+\beta_{3} P_{2}(t)\right. \\
\left.+\beta_{4} P_{3}(t)+\beta_{5} P_{4}(t)+\beta_{6} P_{5}(t)+\beta_{7} J P(t)\right]
\end{gathered}
$$

where $J P(t)$ is a binary variable indicating that an animal is in parity 4 or higher and positive for MAP at time $t$, and $\beta_{7}$ is its parameter.

\section{Calving}

For each animal, calving dates were recorded for each lactation by the herd managers. Calculations were based on time elapsed from the first calving date (Figure 1). The end of the period of interest was the subsequent calving date, if available, or the date of right censoring. If the animal was culled, the culling date was used as the end of time at risk. If the animal was recorded as "do not breed" (DNB) in the herd records, the date of DNB was used as the end of time at risk rather than the culling date; for the purposes of the reproductive model, any reference to culling includes DNB animals. All animals were censored at the end of the study period.

Two models were used to analyze the effect of each of several JD status categories on time to calving, a recurrent event. The first model is a proportional rates model for calving (Lin et al., 2000), with rate function given by

$$
\begin{aligned}
\gamma_{R}(t)= & \gamma_{0 H}(t) \exp \left[\theta_{1} J D_{1}(t)+\theta_{2} J D_{2}(t)+\theta_{3} P_{2}(t)\right. \\
& \left.+\theta_{4} P_{3}(t)+\theta_{5} P_{4}(t)+\theta_{6} P_{5}(t)\right]
\end{aligned}
$$

where $\gamma_{R}(t)$ is the risk of calving at time $t$ and $\gamma_{0 H}(t)$ is an arbitrary and unspecified baseline rate function associated with herd $H$. The binary variables $J D_{1}(t)$, $J D_{2}(t)$, and $P_{i}(t)$ are each coded as in the culling model of Equation 2, thereby continuing to represent ordinal categorical effects, and the regression coefficients $\theta_{1}$ through $\theta_{6}$ corresponding to the main effects therefore have interpretations analogous to those effects in the culling model, measuring the change in calving rates as an animal moves between successive (i.e., adjacent) categories. This rate function makes no explicit assumptions regarding the relationship between the current rate and the past event history beyond the dependence on parity reflected in Equation 3. Valid estimates of the model parameters appearing in Equation 2 may be obtained in the presence of censoring because of culling provided that calving and culling rates are independent given herd, current parity, and current JD status (Lin et al., 2000; Miloslavsky et al., 2004). Because JD status may be positively associated with increasing parity, the variable $J P(t)$, described previously, was added to this model, giving the rate function

$$
\begin{gathered}
\gamma_{R}(t)=\gamma_{0 H}(t) \exp \left[\theta_{1} J D_{1}(t)+\theta_{2} J D_{2}(t)+\theta_{3} P_{2}(t)\right. \\
\left.+\theta_{4} P_{3}(t)+\theta_{5} P_{4}(t)+\theta_{6} P_{5}(t)+\theta_{7} J P(t)\right]
\end{gathered}
$$

where $J P(t)$ is a binary variable indicating that an animal is in parity 4 or higher and positive for MAP at time $t$, and $\theta_{7}$ is its parameter. It is important to note that these models each measure the effect of JD status conditionally upon the level of parity; in other words, the model of Equation 2 characterizes the effect of current JD status on calving rates after adjusting for any effect that past JD status might have on current and past parity. As illustrated in Wolfe and Strawderman (1996), care is needed to avoid fitting models that may inadvertently adjust for the effect of 1 time-dependent variable (i.e., JD status) through the path of another (i.e., parity).

The second model, also a proportional rates regression model, is given by

$$
\gamma_{J D}(t)=\gamma_{1 H}(t) \exp \left[\eta_{1} J D_{1}(t)+\eta_{2} J D_{2}(t)\right],
$$

where $\gamma_{J D}(t)$ is the risk of calving at time $t, \eta_{i}$ measures the direct (i.e., unconditional) effect of the $i$ th 
JD status $J D_{i}(t)$, and $\gamma_{1 H}(t)$ is an arbitrary and unspecified baseline rate function associated with herd $H$. The interpretation of Equation 5 is similar to Equation 3 , describing the calving rate among animals at risk adjusting for herd and JD status only, with JD status coded ordinally as in the models described earlier. However, unlike Equation 3, the effect of JD status on calving rates is now considered to be unconditional and therefore intends to measure the effect of disease regardless of the level of parity. Unfortunately, estimates obtained from this model are also problematic in the presence of censoring by culling. In particular, because culling depends on parity, the failure to control for parity when analyzing the effect of JD status on calving rates actually creates dependent censoring, leading to bias in the estimated model parameters (Miloslavsky et al., 2004).

To characterize the effect of JD status on reproduction rates using a model like that described in Equation 5 , adjustments for the bias induced by dependent censoring need to be made. In cases where the dependent censoring variable can be modeled, as in the case of culling, such bias can be corrected through the use of a weighting factor representing the inverse of the probability of censoring (Robins and Rotnitzky, 1992). An inverse probability of censoring weighted (IPCW) estimator of Miloslavsky et al. (2004) can be used for this purpose. Therefore, we also consider estimating the parameters of the model in Equation 5 using an IPCW-based method of estimation in which animals having a high probability of censoring, such as animals that have not been successfully impregnated early in lactation, are given greater weight to correct for the relative paucity of events.

The implementation of the IPCW estimator of Miloslavsky et al. (2004) in the current context requires survivor function estimates for each animal in each lactation obtained from 2 different culling models. Using Equation 1, the first culling model included stratification by herd and adjusts for both JD status and parity, giving a survivor function for culling of

$$
\begin{aligned}
S_{1 i}\left[t ; R_{i}(t)\right]= & \exp \left\{-\int_{0}^{t} \lambda_{0 H}(u) \exp \left[\beta_{1} J D_{1}(u)\right.\right. \\
& +\beta_{2} J D_{2}(u)+\beta_{3} P_{2}(u)+\beta_{4} P_{3}(u) \\
& \left.\left.+\beta_{5} P_{4}(u)+\beta_{6} P_{5}(u)\right] \mathrm{d} u\right\},
\end{aligned}
$$

where $S_{1 i}\left[t ; R_{i}(t)\right]$ is the survivor function (probability of having not been culled) at time $t ; R_{i}(t)$ refers to the event history for animal $i$ at time $t ; \lambda_{0 H}$ is the baseline culling rate for herd group $H ; i$ indexes animal; $u$ is an increment of time; and all other parameters are defined as for Equation 1. Analogously to Equation 5, the second culling model included only stratification by herd and adjustment for JD status, giving a culling survivor function

$$
\begin{aligned}
S_{2 i}\left[t ; Z_{i}(t)\right]= & \exp \left\{-\int_{0}^{t} \lambda_{2 H}(u) \exp \left[\delta_{1} J D_{1}(u)\right.\right. \\
& \left.\left.+\delta_{2} J D_{2}(u)\right] \mathrm{d} u\right\},
\end{aligned}
$$

where $S_{2 i}\left[t ; Z_{i}(t)\right]$ is the survivor function at time $t ; Z_{i}(t)$ refers to the history of the $J D$ variable for animal $i$ until time $t ; \lambda_{2 H}$ is the baseline culling rate for herd group $H$; and $\delta_{1}$ is the coefficient for the effect of JD status, $J D_{i}(t)$. The ratio $S_{2 i} / S_{1 i}$ was calculated for each cow-lactation-JD status combination (i.e., through time) to define a time-dependent weight $w_{i}(t)$ that was subsequently used to estimate a culling-adjusted calving rate given MAP infection status only. The weight function $w_{i}(t)$ intends to adjust for the resulting effect of dependent censoring, assumed here to be created by omitting those variables from Equation 5 that appear in Equation 3. A second version of $w_{i}(t)$ was also created by including the interaction term $J P(t)$ in Equation 6 , allowing us to consider the effect of the interaction between positive MAP status and parity $>3$ on cullingadjusted calving rates. Estimation of the regression parameter $\eta$ and baseline rate function $\gamma_{1 H}(t)$ are easily accomplished using Cox regression software, provided this software provides the capability of incorporating these time-dependent weights. For example, the baseline rate $\gamma_{1 H}(t)$ is estimated via

$$
\hat{\gamma}_{1 H}(t)=\frac{\sum_{i} w_{i}(t) d N_{i}(t)}{\sum_{i} w_{i}(t) Y_{i}(t) \exp \left[\hat{\eta}_{1} J D_{1}(t)+\hat{\eta}_{2} J D_{2}(t)\right]},
$$

where $d N_{i}(t)$ indicates an incremental increase in the count of events, in this case a calving, $Y_{i}(t)$ indicates that animal $i$ is at risk for calving at time $t$, and $\hat{\eta}_{i}$ is the estimated coefficient for the effect of the JD status variable $J D_{i}(t)$. If censoring were independent of calving given only herd and JD status, $w_{i}(t)$ should approximately be equal to 1 for each $t$ and Equation 8 collapses to the standard estimator for the baseline rate function.

Because management bias may enter the analysis and producers may exert less effort to breed animals known to be infected, time to first insemination is also a variable of interest. An unweighted Andersen-Gill model with control for parity, similar to Equation 2, was fit 
Table 1. Longitudinal data analyzed for the effect of Johne's disease status on time to culling in dairy cattle

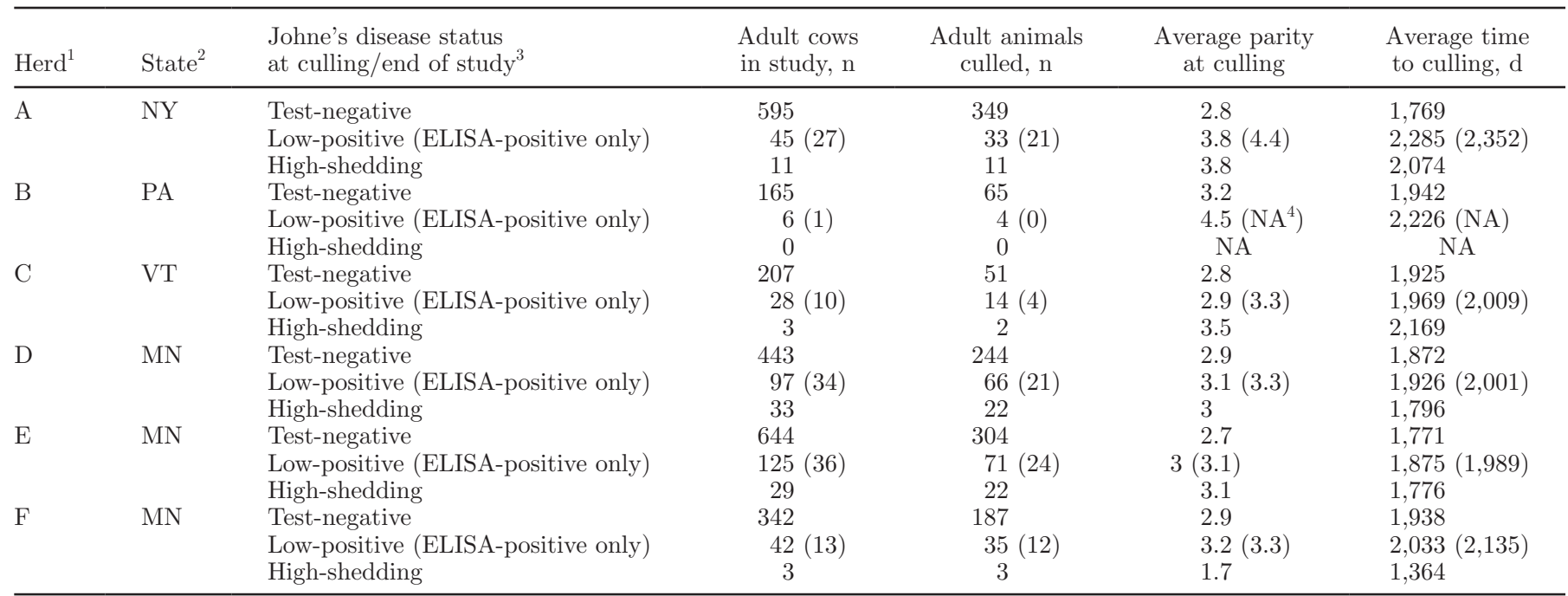

${ }^{1}$ Data for herds A, B, and C from a longitudinal study of 3 dairy herds through the Regional Dairy Quality Management Alliance from 2004 to 2007, visited quarterly to collect individual animal samples and all production, breeding, and health records. Data for herds D, E, and F from the Minnesota Johne's Disease Demonstration Herd Project, which provided annual individual animal samples from each herd from 2000 to 2007. All monthly production, breeding, and culling records were obtained through the DHIA.

${ }^{2} \mathrm{NY}=$ New York; PA = Pennsylvania; VT $=$ Vermont; MN = Minnesota.

${ }^{3}$ Low-positive results are defined as fecal cultures with $\leq 30 \mathrm{cfu} / \mathrm{g}$ of Mycobacterium avium ssp. paratuberculosis, or positive ELISA results, or both.

${ }^{4} \mathrm{NA}=$ not applicable.

to this data. Times were calculated as days elapsed from first calving, with start time being the time of the current calving and end time being the time of the first insemination for that lactation, as shown in Figure 1.

All survival and proportional rate regression analyses were performed with the PHREG procedure in SAS (version 9.2, SAS Institute Inc., Cary, NC). The PHREG procedure provides the ability to compute robust variance estimates (required for use with proportional rate regression models) and time-dependent weights (required for adjusting the estimation of model parameters in a proportional rate regression model in the presence of dependent censoring). Variables were considered significant at the 0.05 level. An effect estimate of less than 0 indicates a lower risk of culling or rate of calving, whereas an effect estimate of greater than 0 indicates a higher risk or rate.

\section{RESULTS}

Data used for the analyses are summarized in Table 1 (culling) and Table 2 (time to calving). Herds in the study provided data on calvings and cullings for between 3.5 and $7 \mathrm{yr}$, including data from cows born within 12 to $15 \mathrm{yr}$ before the end of the study period. Point estimates were similar when analysis was performed on each regional database (RDQMA and JD-
DHP) separately (data not shown), so region was not included in the analysis.

The results of the survival analyses for time to culling are presented in Table 3 . The effect estimate for the low-positive MAP status variable, $J D_{1}(t)$, is significant for all analyses $(P<0.01)$, demonstrating that low-positive animals were more likely to be culled than test-negative animals, even after controlling for parity. The effect estimate for the high-shedding MAP status variable, $J D_{2}(t)$, was not significant, but the interaction term between JD-positive status and parity $>3$ was significant when added. Base culling rates were also observed to vary between herds. The decline observed in culling rates with increasing parity bears comment. In particular, because of the way in which parity is coded, a comparison is made between the indicated parity level and its preceding category. Because time is measured since the first calving date rather than since the beginning of the lactation, animals with higher parity tend to be under observation longer and hence have longer culling times. Culling reasons were provided by herds A, B, and C for 393, 69, and 67 animals, respectively; 143 animals were recorded as having been culled for reproductive reasons, and a further 143 animals were recorded as having been culled for disease reasons, including 8 high-shedding animals recorded as having been culled for clinical JD. Other reasons were given 
Table 2. Longitudinal data analyzed for the effect of Johne's disease status on calving interval in dairy cattle

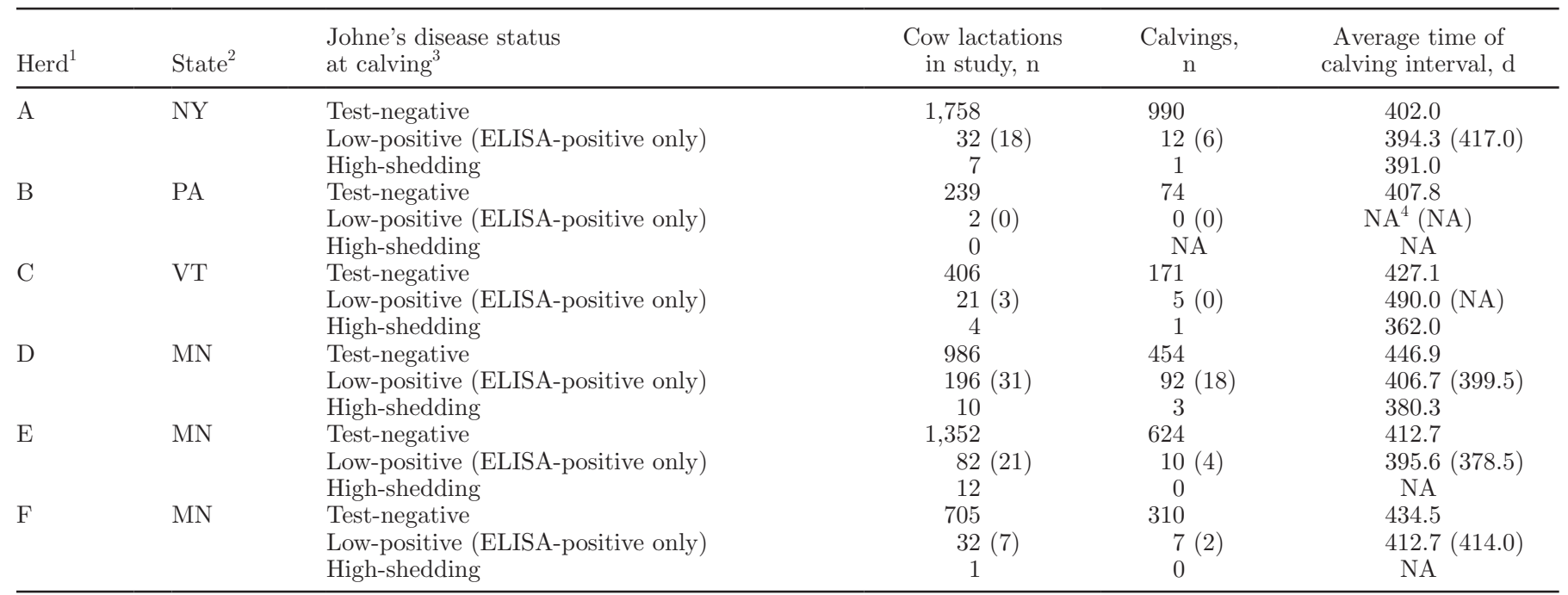

${ }^{1}$ Data for herds A, B, and C from a longitudinal study of 3 dairy herds through the Regional Dairy Quality Management Alliance from 2004 to 2007, visited quarterly to collect individual animal samples and all production, breeding, and health records. Data for herds D, E, and F from the Minnesota Johne's Disease Demonstration Herd Project, which provided annual individual animal samples from each herd from 2000 to 2007. All monthly production, breeding, and culling records were obtained through the DHIA.

${ }^{2} \mathrm{NY}=$ New York; PA = Pennsylvania; VT = Vermont; MN = Minnesota.

${ }^{3}$ Low-positive results are defined as fecal cultures with $\leq 30 \mathrm{cfu} / \mathrm{g}$ of Mycobacterium avium ssp. paratuberculosis, or positive ELISA results, or both.

${ }^{4} \mathrm{NA}=$ not applicable.

for culling a total of 243 animals, including injuries and production.

The results for the proportional rate regression models for time to calving described in the previous section are presented in Table 4. For the models corresponding to Equations 3 and 4, the rate of calving decreased as parity increased (results not shown), with no evidence of a differential effect of JD status for lower versus higher parities. Animals low-positive for MAP (first row of Table 4) were also estimated to have a higher rate of calving compared with test-negative animals. This latter result was also reflected in the results ob- tained for both the unweighted and weighted model fits corresponding to Equation 5, which no longer directly adjusted for parity in the rate model itself. The effect of low-positive status is not statistically significant $(P=0.116)$ when an interaction between parity and JD status is not included in the culling model used to compute the weights, but is borderline significant in the presence of this interaction $(P=0.046)$.

Animals shedding high levels of MAP (the second row of Table 4) exhibited substantial, and more disparate, numerical decreases in the calving rate compared with low-positive animals in each case, with the effect under

Table 3. Results of a proportional hazards model for effect of Johne's disease (JD) status on time to culling ${ }^{1}$

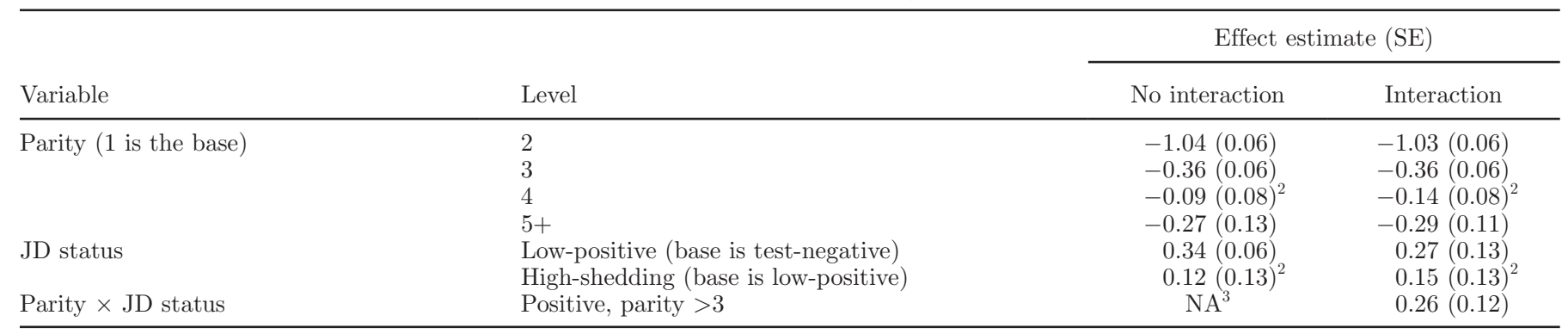

${ }^{1}$ The model takes into account censoring because of end of the study, controls for parity, and is stratified by herd. The model was fit with and without a term for the interaction between JD status and parity $>3$. In the analysis, 6,163 observations were used.

${ }^{2}$ Not significant at the $5 \%$ level.

${ }^{3} \mathrm{NA}=$ not applicable. 
Table 4. Effect estimates from 4 separate sets of proportional rates models for the effect of Johne's disease (JD) status on calving interval, with standard error in parentheses ${ }^{1}$

\begin{tabular}{|c|c|c|c|c|c|c|}
\hline Comparison & $\begin{array}{l}\text { Unweighted effect } \\
\text { estimate for time } \\
\text { to calving } \\
\text { (Equation } 3 \text { ) }\end{array}$ & $\begin{array}{l}\text { Unweighted effect } \\
\text { estimate for } \\
\text { time to calving } \\
\text { with interaction } \\
\text { (Equation 4) }\end{array}$ & $\begin{array}{l}\text { Unweighted effect } \\
\text { estimate for } \\
\text { time to calving } \\
\text { (Equations } 5 \text { and } 8 \text { ) }\end{array}$ & $\begin{array}{c}\text { Weighted effect } \\
\text { estimate for } \\
\text { time to calving } \\
\text { (Equations } 5 \text { and } 8 \text { ) }\end{array}$ & $\begin{array}{c}\text { Weighted effect } \\
\text { estimate for } \\
\text { time to calving } \\
\text { with interaction } \\
\text { (Equations } 5 \text { and } 8 \text { ) }\end{array}$ & $\begin{array}{l}\text { Effect estimate } \\
\text { for time to } \\
\text { first } \\
\text { insemination }\end{array}$ \\
\hline $\begin{array}{l}\text { Low-positive } \\
\text { (test-negative is base) } \\
\text { High-shedding }\end{array}$ & $0.18(0.07)$ & $0.17(0.08)$ & $0.14(0.06)$ & $0.20^{2}(0.12)$ & $0.23(0.12)$ & $0.01^{2}(0.06)$ \\
\hline
\end{tabular}

${ }^{1}$ The unweighted model for Equation 3 adjusts for JD status, parity, and herd (through stratification). The unweighted model for Equation 4 adjusts for JD status, parity, and herd (through stratification) and the interaction between test-positive status and parity $>3$. The unweighted model for Equation 5, using the baseline function of Equation 8 with weights set equal to 1, adjusts only for JD status and herd (through stratification), not including parity. The weighted model for Equation 5, using the baseline function of Equation 8, controls for both the probability of culling and parity through weighting, with or without the interaction between test-positive status and parity $>3$, controlling for herd through stratification. The results of an unweighted proportional rates model based on time to first insemination, controlling for parity and stratified by herd, are also included. In the analysis, 6,163 observations were included.

${ }^{2}$ Not significant at the $5 \%$ level.

the weighted model fit corresponding to Equation 5 being both the strongest and the most statistically significant. Comparison of the effect estimates for the fits corresponding to Equation 5 show that the weighted estimate is more than twice the unweighted estimate for high-shedding animals. In our view, these results clearly illustrate 2 features of the proposed methodology. First, a comparison of the differences in effect estimates obtained for the unweighted and weighted fits corresponding to the model of Equation 5 demonstrates the effect of dependent censoring that results from a failure to control for the effect that parity has on culling (i.e., the dependent censoring variable). Here, we further observe that inclusion of the interaction term $J P(t)$ did not qualitatively or quantitatively affect the estimates for any model. Second, a comparison of the model fits for Equation 4 and the weighted fits corresponding to Equation 5 illustrates the differences in interpretation of the regression coefficients in models that directly, versus indirectly, adjust for parity when analyzing the effect of JD status (Wolfe and Strawderman, 1996). In particular, the regression coefficient in Equation 4 is attenuated, a reflection of the fact that a model that directly adjusts for both parity and JD status is capturing the effect of JD status on calving rates that remains after adjusting for any effect it may have had on past and current parity. Results of the analysis for time to first insemination (Table 4) further showed that time to first insemination was not significantly related to JD status.

\section{DISCUSSION}

The models presented above analyzed the relationship between JD status (as defined by MAP shedding and ELISA results) and both the risk of culling and calving rates. The results of the model for time to culling were easily influenced by producer decisions. In all herds, producers were interested in controlling or eradicating JD and were informed of test results when available. Based on culling reasons given for high-shedding animals, in herds $\mathrm{A}$ and $\mathrm{C}$, JD status was the most common reason stated. Thus, the results likely show a combination of both biological and producer-decision effects, with the latter influenced by expert advice.

Beyond the desire to control the spread of JD, producers often cull animals because of low or decreased milk production. The authors have previously observed, with the same data set, a significant relationship between JD status and decreasing milk production (Smith et al., 2009). Low-positive and test-negative animals did not have significantly different average daily milk production levels in that analysis, whereas milk production in low-positive animals did decrease over time faster than test-negative controls. High-shedding animals, when compared with all other animals, were observed to have both a significantly lower average daily milk production and a significantly greater monthly decrease in milk production. This decreased milk production observed in MAP-positive animals would be expected to lead to the increased rate of culling in MAP-positive animals observed in the present study, as an intervening variable on the causal pathway between JD and culling. The increased culling rate could also be related to other effects of JD (including the hypothesized decreased calving rates) or to the producer's desire to control JD through culling. The former has been observed previously; fecal culture-positive cows were observed to be culled for infertility at a higher rate than culturenegative cows (Merkal et al., 1975). In these data, the 
latter was also known to play a role because the herds participate in a JD control program that recommends culling of high-positive animals.

Inclusion of a term to capture the interaction between JD status and age (via the proxy of parity) was found to be necessary. However, the use of a saturated interaction model would have required 8 additional terms in the full model. In addition to creating serious challenges in model interpretation (particularly given our use of an ordinal coding strategy), the presence of relatively sparse data available for high-shedding animals raised questions of feasibility. Our decision to use a simpler interaction term that differentiated older, positive animals from the rest was motivated by a preliminary data analysis using a simple 4-level variable that classified animals according to JD status ("any" vs. not) and age ("parity $>3$ " vs. not) and that suggested that the strongest effect was in these animals (results not shown). Although this interaction term was found to be statistically significant in the full culling model of Equation 3, its inclusion did not qualitatively change the results of the culling model or the weighted calving models.

Because of the positive dependency between calving, culling, parity, and JD status, indicated by the discrepancy in the results presented in Table 4 and the large proportion of animals culled for reasons of reproductive problems, a correction to the standard proportional rates model was required to more appropriately analyze the effect of JD status on reproduction. With the inverse probability of censoring weighted estimator approach of Miloslavsky et al. (2004), a direct estimate of the marginal effect of JD status can be obtained. Other methods, such as frailty models (Rondeau et al., 2007), might have been used instead to deal with the possibility of dependent censoring as well as the dependence between multiple calvings in the same animal; however, such methods may yield less interpretable estimates of the effect of JD status. In this study, the difference between the inverse probability of censoring weighted estimator-corrected (i.e., weighted) and basic (i.e., unweighted) models was found to be relatively small for the case of all low-positive animals and substantially larger for high-shedding animals. For both levels of JD status, the weighted analysis was also found to be more conservative, resulting in larger standard errors. The addition of an interaction term to capture the synergy between age and test-positive status did not qualitatively change the results of any model. Thus, for example, one can conclude that a synergistic relationship between age and JD status on culling rates is unlikely to be responsible for the observed increase in calving intervals in high-shedding cows obtained when comparing the weighted and unweighted model fits corresponding to Equation 5.

There were possible biases in this study introduced by the method of measurement available. Because reproduction was measured based on the proxy of calving intervals rather than time to conception, some animals censored for the end of study may have conceived but not reached calving. Animals were also considered at risk for calving, in this model, during the voluntary waiting period, breeding, and early gestation. That was not precisely true because animals were at risk of calving only in the end of gestation. It was assumed that no bias arose from ignoring end-of-study censoring, the voluntary waiting period, and gestation time; all these should have been unassociated with JD status and should not have affected the estimates. This left only the effects of JD status on breeding, which included both producer decisions and biological effects on conception. However, a change in JD status may occur between conception and calving; in this analysis, the event of interest would be falsely attributed to the higher JD status. Future studies may avoid these issues by recording conception dates when collecting data, allowing for a more direct analysis. It would also be of interest to consider the effect of latent infection on production parameters, but such an analysis is not possible within the scope of these data.

The categorization of JD status could also lead to biased estimates; animals were assumed to be truly positive or negative despite the imperfect specificity and sensitivity of the diagnostic tests. Any passive shedders (animals with positive fecal samples that were not infected) would confuse the categories used, making differences difficult to detect. False-positive results would also bias the estimates toward the null. Further analysis of the question within research herds, with tissue culture of animals on slaughter to ensure proper categorization, may lead to stronger or more significant findings.

This study also combined 2 separate databases, which were collected for similar purposes but with different methods. The point estimates for the effects of JD status on both culling and reproduction were similar if the 2 databases were analyzed separately; thus, we concluded that combining the data sets, leading to improved precision of all estimates, was appropriate.

Milk production level is known to influence calving rates (Lucy, 2001), and the prevailing understanding is that high milk production is correlated with decreased calving rates. We did not include this in our analysis, however, to capture the full effect of JD status on calving rates. In our study, high-shedding cows tended to have lower calving rates than others, but previous analysis 
of the RDQMA database showed that high-shedding cows had significantly lower milk production compared with all other cows (Smith et al., 2009). Therefore, milk production could be considered an intervening variable in the pathway between JD status and calving rates rather than a confounding variable. Calving season may also affect reproduction, but calving season was found not to be associated with JD status in our data, so we did not include it in the model.

Our data also showed that low-positive animals had higher calving rates than test-negative animals. This agrees with an earlier published study (Lombard et al., 2005), which found that animals with a strong-positive ELISA had significantly fewer days open than ELISAnegative animals. However, Lombard et al. (2005) were not able to distinguish between stages of JD because fecal culture results were not provided in that study. Marcé et al. (2009) also found higher calving rates among ELISA-positive animals, but the decrease of the effect with increasing parity suggests that disease progression may reverse the effect. Our results suggest that movement from low-positive to high-shedding of MAP was associated with decreased calving rates, which would agree with the findings of Marcé et al. (2009). The opposing effects of JD in low-positive and high-shedding animals, also observed to some extent by Gonda et al. (2007), may explain the failure of previous studies (de Lisle and Milestone, 1989; McNab et al., 1991) to find an effect in positive animals, especially because controlling for the effect of JD on culling proved to be necessary.

Decreased calving rates can be an expensive loss for dairy herds, but the cause is uncertain. They could possibly indicate a biological cause, such as the postulated effect of clinical JD leading to a negative energy balance, which can lead to an anovulatory state (Butler et al., 2006), significantly delaying time to conception (Butler, 2003). However, it was not possible, with these data, to determine that the effect of the disease on calving was attributable to biological effects of disease rather than producer decisions. Not every herd in this study recorded when an animal was labeled DNB, and even herds that did record it may have done so sporadically. As such, animals that were not at risk for conception would have been mistakenly included in the data set. Producers may also have bred MAP-positive animals less frequently than MAP-negative animals, with an unconscious or unrecorded bias, artificially decreasing their risk of conception. Raizman et al. (2007) found no association between JD status and days open, but did find that nonpregnant animals with positive fecal cultures were bred significantly fewer times than testnegative animals. In the same way, animals that were high milk producers (such as latently infected animals, in this model) may have been bred more intensively, with more inseminations than lower-producing animals. For this reason, we examined the time to first insemination as a proxy of producer effort. However, time to first insemination was not significantly affected by JD status, which suggests a biological cause for the observed effect on calving intervals.

In conclusion, survival analysis indicated that detectable infection with MAP resulted in increased culling rates. We also observed an increased calving interval in animals shedding high levels of MAP compared with low-positive animals.

\section{ACKNOWLEDGMENTS}

The USDA (Cooperative State Research, Education and Extension Service, Washington, DC) Award Number 2008-35204-04627 provided funding for this study, as did the USDA-Agricultural Research Service (Agreements 58-1265-3-155, 58-1265-3-156, 58-1265-3158, and 58-1265-4-020) for the Regional Dairy Quality Management Alliance (RDQMA; Beltsville, MD) and the Johne's Disease Integrated Program (JDIP; University Park, PA; USDA contract 45105).

\section{REFERENCES}

Abbas, B., H. P. Rienmann, and D. W. Hird. 1983. Diagnosis of Johne's disease (paratuberculosis) in northern California cattle and a note on its economic significance. Calif. Vet. 8:19-24.

Allore, H. G., L. D. Warnick, J. Hertl, and Y. T. Grohn. 2001. Censoring in survival analysis: A simulation study of the effect of milk yield on conception. Prev. Vet. Med. 49:223-234.

Andersen, P. K., and R. D. Gill. 1982. Cox's regression model counting process: A large sample study. Ann. Stat. 10:1100-1120.

Buergelt, C. D., and J. R. Duncan. 1978. Age and milk production data of cattle culled from a dairy herd with paratuberculosis. J. Am. Vet. Med. Assoc. 173:478-480.

Butler, S. T., S. H. Pelton, and W. R. Butler. 2006. Energy balance, metabolic status, and the first postpartum ovarian follicle wave in cows administered propylene glycol. J. Dairy Sci. 89:2938-2951.

Butler, W. R. 2003. Energy balance relationships with follicular development, ovulation and fertility in postpartum dairy cows. Livest. Prod. Sci. 83:211-218.

Clarke, C. J. 1997. The pathology and pathogenesis of paratuberculosis in ruminants and other species. J. Comp. Pathol. 116:217-261.

Collins, M. T. 2003. Paratuberculosis: Review of present knowledge. Acta Vet. Scand. 44:217-221.

Cox, D. R. 1972. Regression models and life-tables (with discussion). J. R. Stat. Soc. B 34:187-220.

de Lisle, G. W., and B. A. Milestone. 1989. The economic impact of Johne's disease in New Zealand. Pages 41-45 in Johne's Disease, Current Trends in Research Diagnosis and Management. A. R. Milner and P. R. Wood, ed. CSIRO Publications, Melbourne, Australia.

Dorshorst, N. C., M. T. Collins, and J. E. Lombard. 2006. Decision analysis model for paratuberculosis control in commercial dairy herds. Prev. Vet. Med. 75:92-122.

Ferrouillet, C., S. J. Wells, W. L. Hartmann, S. M. Godden, and J. Carrier. 2009. Decrease of Johne's disease prevalence and incidence in six Minnesota, USA, dairy cattle herds on a long-term management program. Prev. Vet. Med. 88:128-137. 
Fourichon, C., H. Seegers, and X. Malher. 2000. Effects of disease on reproduction in the dairy cow: A meta-analysis. Therio. 53:17291759.

Gonda, M. G., Y. M. Chang, G. E. Shook, M. T. Collins, and B. W. Kirkpatrick. 2007. Effect of Mycobacterium paratuberculosis infection on production, reproduction, and health traits in US Holsteins. Prev. Vet. Med. 80:103-119.

Gröhn, Y. T., S. W. Eicker, V. Ducrocq, and J. A. Hertl. 1998. Effect of diseases on the culling of Holstein dairy cows in New York State. J. Dairy Sci. 81:966-978.

Johnson-Ifearulundu, Y. J., J. B. Kaneene, D. J. Sprecher, J. C. Gardiner, and J. W. Lloyd. 2000. The effect of subclinical Mycobacterium paratuberculosis infection on days open in Michigan, USA, dairy cows. Prev. Vet. Med. 46:171-181.

Lin, D. Y., L. J. Wei, I. Yang, and Z. Ying. 2000. Semiparametric regression for the mean and rate functions of recurrent events. J. R. Stat. Soc. B 52:711-730.

Lombard, J. E., F. B. Garry, B. J. McCluskey, and B. A. Wagner. 2005. Risk of removal and effects on milk production associated with paratuberculosis status in dairy cows. J. Am. Vet. Med. Assoc. 227:1975-1981.

Lu, Z., R. M. Mitchell, R. L. Smith, J. S. Van Kessel, P. P. Chapagain, Y. H. Schukken, and Y. T. Grohn. 2008. The importance of culling in Johne's disease control. J. Theor. Biol. 254:135-146.

Lucy, M. C. 2001. Reproductive loss in high-producing dairy cattle: Where will it end? J. Dairy Sci. 84:1277-1293.

Marcé, C., F. Beaudeau, N. Bareille, H. Seegers, and C. Fourichon 2009. Higher non-return rate associated with Mycobacterium avium subspecies paratuberculosis infection at early stage in Holstein dairy cows. Theriogenology 71:807-816.

McNab, W. B., A. H. Meek, S. W. Martin, and J. R. Duncan. 1991. Associations between dairy production indices and lipoarabinomannan enzyme-immunoassay results for paratuberculosis. Can. J. Vet. Res. 55:356-361.

Merkal, R. S., A. B. Larsen, and G. D. Booth. 1975. Analysis of the effects of inapparent bovine paratuberculosis. Am. J. Vet. Res. 36:837-838.

Miloslavsky, M., S. Keles, and M. J. van der Laan. 2004. Recurrent events analysis in the presence of time-dependent covariates and dependent censoring. J. R. Stat. Soc. B 66:239-257.

Nielsen, S. S. 2008. Transitions in diagnostic tests used for detection of Mycobacterium avium ssp. paratuberculosis infections in cattle. Vet. Microbiol. 132:274-282.

Nielsen, S. S., and N. Toft. 2008. Ante mortem diagnosis of paratuberculosis: A review of accuracies of ELISA, interferonassay, and faecal culture techniques. Vet. Microbiol. 129:217235.

Ott, S. L., S. J. Wells, and B. A. Wagner. 1999. Herd-level economic losses associated with Johne's disease on US dairy operations. Prev. Vet. Med. 40:179-192.
Pradhan, A. K., J. S. Van Kessel, J. S. Karns, D. R. Wolfgang, E. Hovingh, K. A. Nelen, J. M. Smith, R. H. Whitlock, T. L. Fyock, S. Ladely, P. J. Fedorka-Cray, and Y. H. Schukken. 2009. Dynamics of endemic infectious diseases of animal and human importance on three dairy herds in the Northeastern US. J. Dairy Sci. 92:18111825.

Raizman, E. A., J. P. Fetrow, S. J. Wells, S. M. Godden, M. J. Oakes, and G. Vazquez. 2007. The association between Mycobacterium avium ssp. paratuberculosis fecal shedding or clinical Johne's disease and lactation performance on two Minnesota, USA dairy farms. Prev. Vet. Med. 78:179-195.

Robins, J. R., and A. Rotnitzky. 1992. Recovery of information and adjustment for dependent censoring using surrogate markers. Pages 24-33 in AIDS Epidemiology: Methodological Issues. Birkhauser, Boston, MA.

Rondeau, V., S. Mathoulin-Pelissier, H. Jacqmin-Gadda, V. Brouste, and P. Soubeyran. 2007. Joint frailty models for recurring events and death using maximum penalized likelihood estimation: Application on cancer events. Biostatistics 8:708-721.

Smith, R. L., Y. T. Grohn, A. K. Pradhan, R. H. Whitlock, J. S. Van Kessel, J. M. Smith, D. R. Wolfgang, and Y. H. Schukken. 2009. A longitudinal study for the impact of Johne's disease status on milk production in individual cows. J. Dairy Sci. 92:2653-2661.

USDA-APHIS-VS-NAHMS. 2007. Johne's Disease on U.S. Dairy Operations. Report \#N521.0408. USDA-APHIS-VS-NAHMS, Fort Collins, CO.

van Roermund, H. J. W., D. Bakker, P. T. J. Willemsen, and M. C. M. de Jong. 2007. Horizontal transmission of Mycobacterium avium ssp. paratuberculosis in cattle in an experimental setting: Calves can transmit the infection to other calves. Vet. Microbiol. 122:270-279.

van Schaik, G., C. R. Rossiter, S. M. Stehman, S. J. Shin, and Y. H. Schukken. 2003. Longitudinal study to investigate variation in results of repeated ELISA and culture of fecal samples for Mycobacterium avium subsp. paratuberculosis in commercial dairy herds. Am. J. Vet. Res. 64:479-484.

Wells, S. J., B. A. Wagner, C. J. Lindeman, and T. L. Fyock. 2002. Evaluation of bacteriologic culture of pooled faecal samples for detection of Mycobacterium paratuberculosis. Am. J. Vet. Res. 63:1207-1211.

Whitlock, R. H., S. J. Wells, R. W. Sweeney, and T. J. Van. 2000. ELISA and fecal culture for paratuberculosis (Johne's disease) Sensitivity and specificity of each method. Vet. Microbiol. $77: 387-398$.

Wolfe, R. A.. and R. L. Strawderman. 1996. Logical and statistical fallacies in the use of Cox regression models. Am. J. Kidney Dis. $27: 124-129$. 